\title{
Producción de tomate (Solanum lycopersicum) en invernadero: comparación agronómica entre tipos de tomate
}

\section{Greenhouse tomato (Solanum lycopersicum) production: agronomic comparison between types of tomato}

\author{
José Eladio Monge Pérez ${ }^{1}$ \\ Universidad de Costa Rica, Costa Rica \\ Michelle Loría Coto ${ }^{2}$ \\ Universidad Estata a Distancia, Costa Rica
}

\begin{abstract}
Resumen. Se evaluaron cuatro tipos de tomate (uva, cherry, cocktail, y gordo), producidos bajo invernadero, a nivel cuantitativo (10 variables). Los datos muestran una amplia variabilidad entre los tipos de tomate en cuanto a edad al inicio de la cosecha (64.8 - 76.5 días después de trasplante), número de frutos por racimo (4.46 - 22.05), número de lóculos por fruto $(2.00-6.21)$, peso del fruto $(12.41-126.80 \mathrm{~g})$, rendimiento $(28.17-51.18$ ton/ha), firmeza del fruto $(21.34-47.13 \mathrm{~N})$, porcentaje de sólidos solubles totales $\left(4.41-8.08{ }^{\circ} \mathrm{Brix}\right)$, pH del jugo del fruto (3.73 - 4.02$)$, índice de sabor $(70,83$ - 131,32), y resultados de la prueba de degustación (1.32 - 2.53 en una escala de 0 a 4$)$. Se concluye que, en comparación con los otros tipos de tomate evaluados, los tomates gordos iniciaron la cosecha en forma más tardía, produjeron menos frutos por racimo, presentaron ,más lóculos por fruto, tuvieron frutos de más peso, produjeron un mayor rendimiento; y sus frutos fueron más firmes, tuvieron un menor porcentaje de sólidos solubles totales, y obtuvieron los peores resultados en la prueba de degustación. Con respecto a los tomates uva, cocktail y cherry, las únicas diferencias significativas entre ellos se presentaron en el número de frutos por racimo, la firmeza del fruto, el porcentaje de sólidos solubles totales, el pH del jugo del fruto, y el índice de sabor.
\end{abstract}

Palabras clave. Solanum lycopersicum, calidad, rendimiento, ${ }^{\circ}$ Brix, peso del fruto

\begin{abstract}
Four tomato types (grape, cherry, cocktail and round) grown under greenhouse conditions were evaluated at quantitative level (10 variables). Data show ample variability between tomato types with respect to the following variables: days to harvest (64.8 - 76.5 days after transplant), number of fruits per cluster $(4,46-22,05)$, number of locules per fruit $(2,00-6,21)$, fruit weight $(12.41-126.80 \mathrm{~g})$, yield $(28.17-51,18 \mathrm{ton} / \mathrm{ha})$, fruit firmness $(21.34-47.13$ $\mathrm{N})$, percentage of total soluble solids $\left(4.41-8.08{ }^{\circ} \mathrm{Brix}\right)$, fruit juice $\mathrm{pH}(3.73-4.02)$, flavor index $(70.83-131.32)$, and sensorial test (1.32 - 2.53 in a 0 to 4 scale). It is concluded that, compared to the other tomato types in this evaluation, round tomatoes reach harvest later, produce a higher yield, bear less fruits per cluster and their fruits have more locules, higher weight and firmness, a lower percentage of total soluble solids, and the worst results in the sensorial test. With respect to grape, cocktail and cherry tomatoes, the only significant differences between them were in the number of fruits per cluster, fruit firmness, percentage of total soluble solids, fruit juice $\mathrm{pH}$, and flavor index.
\end{abstract}

Keywords. Solanum lycopersicum, quality, yield, ${ }^{\circ}$ Brix, fruit weight

${ }^{1}$ Ingeniero agrónomo. Estación Experimental Agrícola Fabio Baudrit Moreno y Sede de Guanacaste, Universidad de Costa Rica, Costa Rica. Dirección electrónica: melonescr@yahoo.com.mx

${ }^{2}$ Economista agrícola. Escuela de Ciencias Exactas y Naturales, Universidad Estatal a Distancia, Costa Rica. Dirección electrónica: michelle_loria@yahoo.com 


\section{Introducción}

El tomate es una de las hortalizas que se cultiva en mayor extensión en el mundo. En el caso de la producción bajo invernadero, se cultivan principalmente genotipos de tomate de crecimiento indeterminado, de diferentes tamaños de fruto: pequeños ('cherry' y uva), medianos ('cocktail'), y grandes (gordos, para racimo y 'saladette') (Castellanos, 2009).

La selección del genotipo adecuado es un aspecto clave en todo proyecto de producción hortícola, pues cada uno de ellos presenta diferentes características de crecimiento de la planta, cualidades del fruto, tolerancia a plagas y enfermedades, y respuesta a las condiciones ambientales. De la misma forma, la escogencia del tipo de tomate a producir es muy importante, pues cada uno de ellos está dirigido a un nicho de mercado específico, que varía en cuanto a volumen de la demanda, precio, criterios de calidad, tipo de empaque, entre otros.

En el cultivo de tomate, la calidad de sus frutos depende de sus características físicas, tales como el color, la firmeza, el tamaño o la forma; de sus cualidades químicas, que incluye el contenido de sólidos solubles, el $\mathrm{pH}$, la acidez titulable y la relación azúcares/ácidos; y de sus componentes nutricionales (aporte de vitaminas y minerales) (Castellanos, 2009).

En el tomate, el sabor depende en gran medida por la concentración de fructosa, glucosa, ácido cítrico y ácido málico (Causse, Buret, Robini, y Verschave, 2003; Cebolla-Cornejo, y otros, 2011; Fernández-Ruiz, y otros, 2004; Shirahige, y otros, 2009; Alonso, García-Aliaga, García-Martínez, Ruiz, y Carbonell-Barrachina, 2009).

El cultivo bajo ambiente protegido modifica muchas veces la respuesta de los cultivos, en relación a lo que sucede en la producción a campo abierto, debido a los cambios en las variables climáticas. Por esta razón se recomienda implementar ensayos con diferentes tipos de tomate y genotipos, para escoger los que mejor se comportan bajo ambiente protegido en cada zona (Castellanos, 2009). Se han realizado diversas investigaciones sobre este tema en varios países (Moricz, s.f.; Hernández, 1987; Lorca, 1997; Santiago, Mendoza, y Borrego, 1998; Campiño y Puerto, 2000; Borrego, y otros, 2001; Grijalva-Contreras, Macías-Duarte, ValenzuelaRuiz, y Robles-Contreras, Productivity and fruit quality in tomatoes varieties under greenhouse conditions in the Northwest of Mexico, 2004; Shirahige, y otros, 2009; Ardila, Fischer, y Balaguera-López, 2011; Grijalva-Contreras, Macías-Duarte, y Robles-Contreras, 2011; Montenegro, 2012; Pérez, Albarracín, Moratinos, y Zapata, 2012; Monge-Pérez, 2014; MongePérez, 2015; Monge-Pérez, 2016).

El objetivo de esta investigación fue evaluar la calidad y producción de cuatro tipos de tomate cultivados bajo ambiente protegido, en Alajuela, Costa Rica.

\section{Materiales y métodos}

El ensayo se realizó en condiciones hidropónicas, en el invernadero de Hortalizas de la Estación Experimental Agrícola Fabio Baudrit Moreno (EEAFBM), la cual está localizada en Barrio San José de Alajuela, Costa Rica, a una altitud de 883 m.s.n.m. Dicho invernadero es marca Richel (Francia), y es de tipo multicapilla. Se sembraron 60 genotipos de tomate (Solanum lycopersicum L.), que representan cuatro tipos diferentes de tomate: 'cherry' (25 genotipos), 'cocktail' (3 genotipos), gordo (17 genotipos), y uva (15 genotipos) (tabla 1 ).

Los criterios utilizados para establecer estos tipos de tomate se basan en la clasificación comercial que usan las empresas productoras de semillas de tomate, y fueron los siguientes: 'cherry': fruto de forma redondeada o redondoalargada, con un peso menor o igual a 22 g; 
Tabla 1

Genotipos de tomate utilizados en la investigación

Tipo de tomate

\begin{tabular}{|c|c|}
\hline $\begin{array}{l}\text { 'Cherry' } \\
(n=25)\end{array}$ & $\begin{array}{l}\text { Tangerino (United Genetics); } 8263 \text { (Nirit); Amarillo (DP Seeds); Mini Charm (DP } \\
\text { Seeds); Mini Star (DP Seeds); Cherry } 1 \text { (Green Seeds); Cherry } 2 \text { (Green Seeds); Cherry } \\
3 \text { (Green Seeds); Cherry } 4 \text { (Green Seeds); Baby Cakes (DP Seeds); Grande Charm (DP } \\
\text { Seeds); Sweet Cherry (Western Hybrid); BHN-624 (Johnny's); BHN-968 (Johnny's); } \\
\text { Black Cherry (Johnny's); Favorita (Johnny's); Gold Nugget (Johnny's); Matt's Wild } \\
\text { Cherry (Johnny's); Sakura (Johnny's); Sun Cherry (Johnny's); Sun Gold (Johnny's); } \\
\text { Tomatoberry Garden (Johnny's); Washington Cherry (Johnny's); White Cherry (Jo- } \\
\text { hnny's); Yellow Mini (Johnny's) }\end{array}$ \\
\hline $\begin{array}{l}\text { 'Cocktail' } \\
(n=3)\end{array}$ & 72919 (Nirit); Giovanni (Nirit); Z-484 (Nirit) \\
\hline $\begin{array}{l}\text { Gordo } \\
(n=17)\end{array}$ & $\begin{array}{l}\text { Endeavour (Rijk Zwaan); 68-39-179 (Pandia); 68-39-177 (Pandia); VT-4 (Namdhari); } \\
\text { Furia (Green Seeds); Aida (Nirit); Metropol (Nirit); Otelo (Nirit); Tatiana (Nirit); } 7026 \\
\text { (Nirit); NP-498 (Tan Nong Phat); IT-01-27 (DP Seeds); Pamella (DP Seeds); Sacramen- } \\
\text { to (DP Seeds); NS-537 (Namdhari); Tropic (Green Seeds); Criollo (Rijk Zwaan) }\end{array}$ \\
\hline $\begin{array}{l}\text { Uva } \\
(n=15)\end{array}$ & $\begin{array}{l}\text { Grappolino (Nirit); Lunico (Nirit); Hy Brix (DP Seeds); Pink Beauty (DP Seeds); Red } \\
\text { Grape (DP Seeds); Uva Roja (DP Seeds); Zuchello (DP Seeds); Dolce Vita (DP Seeds); } \\
\text { Red Scorpion (DP Seeds); Sweet Grape (Western Hybrid); Five Star Grape (Johnny's); } \\
\text { Golden Sweet (Johnny's); Red Pearl (Johnny's); Sakura Honey (Johnny's); Smarty (Jo- } \\
\text { hnny's) }\end{array}$ \\
\hline
\end{tabular}

Nota. Monge y Loría, 2018, a partir de los genotipos utilizados. El nombre entre paréntesis corresponde a la empresa productora de la semilla de cada genotipo.

uva: fruto de forma elipsoide o cilíndrica, con un peso menor o igual a 22 g; 'cocktail': frutos redondeados o elipsoides, con un peso entre 22.1 y 59.9 g; gordo: frutos redondeados, o ligeramente achatados, o redondo-alargados, con un peso igual o mayor a $60 \mathrm{~g}$.

En razón la gran cantidad de genotipos por evaluar, se realizaron dos siembras diferentes. El primer grupo de genotipos se trasplantó el 4 de setiembre de 2012, y el segundo grupo se trasplantó los días 25 de setiembre, 2 de octubre y 9 de octubre de 2012, según el desarrollo de las plántulas de cada híbrido.
El cultivo se realizó en sacos de fibra de coco, de $1 \mathrm{~m}$ de largo, $20 \mathrm{~cm}$ de ancho y 15 $\mathrm{cm}$ de altura. La distancia de siembra fue de $25 \mathrm{~cm}$ entre plantas, y de $1.54 \mathrm{~m}$ entre hileras, para una densidad de 2.60 plantas $/ \mathrm{m}^{2}$. Para la sujeción de las plantas, se utilizaron mallas plásticas, ubicadas una a cada lado de cada hilera de plantas. Se eliminaron todos los tallos secundarios, de manera que las plantas crecieron únicamente a partir del tallo principal.

La cosecha inició el 16 de noviembre de 2012 para ambos grupos de genotipos, y la evaluación de los frutos se llevó a cabo hasta el 8 de enero 
de 2013 para el primer grupo, y hasta el 22 de enero de 2013 para el segundo grupo. Para ambos grupos, la producción se extendió más allá del periodo evaluado, pero no fue posible cuantificar dicha producción adicional.

El combate de plagas se basó en un sistema de manejo integrado, mediante el uso de trampas pegajosas de colores amarillo y azul, uso de hongos entomopatógenos, y aplicaciones de plaguicidas basadas en los monitoreos diarios. Además, se utilizó un programa de fertirrigación a partir de sales solubles, validado previamente para la producción comercial de tomate. El fertirriego se realizó en forma diaria, entre las 7:00 a.m. y las 4:00 p.m., con un riego suministrado cada 60 minutos en ese periodo.

A partir de los descriptores internacionales para el cultivo de tomate (IPGRI, 1996), se seleccionaron las siguientes variables a evaluar:

1. Edad al inicio de la cosecha (ddt): se obtuvo al registrar el día de inicio de la cosecha en cada parcela, según la fecha de trasplante y se obtuvo el promedio.

2. Número de frutos por racimo: se obtuvo al seleccionar 10 racimos por parcela, se contó el número de frutos por racimo y se calculó el promedio.

3. Número de lóculos del fruto: se obtuvo por observación visual, luego de realizar un corte transversal a 10 frutos por parcela y se obtuvo el promedio.

4. Peso del fruto (g): se midió el peso individual de 20 frutos por cada parcela y se calculó el promedio.

5. Rendimiento (ton/ha): se estimó el peso de los frutos de tomate producidos en una hectárea, a partir del rendimiento por planta y la densidad de siembra. El rendimiento se evaluó hasta los $126 \mathrm{ddt}$ en la primera prueba, y entre los $105 \mathrm{y}$ $119 \mathrm{ddt}$ en la segunda prueba.
6. Firmeza del fruto $(\mathrm{N})$ : se midió la firmeza de 20 frutos por cada parcela y se obtuvo el promedio.

7. Porcentaje de sólidos solubles totales ( ${ }^{\circ}$ Brix): se midió el porcentaje de sólidos solubles totales de 20 frutos por cada parcela, y se calculó el promedio.

8. $\mathrm{pH}$ del jugo del fruto: se cuantificó el $\mathrm{pH}$ del jugo de 10 frutos de cada parcela, y se obtuvo el promedio.

9. Índice de sabor $\left[\left({ }^{\circ} \mathrm{Brix}\right)^{*}(\mathrm{pH})^{2}\right]$ : se obtuvo al multiplicar el porcentaje de sólidos solubles totales promedio por el cuadrado del valor del $\mathrm{pH}$ promedio, y para cada parcela se calculó el promedio.

10. Prueba de degustación de sabor: con algunos genotipos (13 'cherry'; 9 uva; 2 gordo; $y 1$ 'cocktail'), se realizó una prueba de degustación, también llamado análisis sensorial (Moricz, s.f.; Pillsbury, Maynard, y Hayes, s.f.), entre 61 personas, en la cual se realizó una evaluación cuantitativa de esta característica, para lo cual se empleó la siguiente escala 'hedonística' de cinco puntos: $0=$ pésimo; $1=$ malo; $2=$ regular; $3=$ bueno; 4 = muy bueno. Para cada tipo de tomate se obtuvo un promedio.

El peso de los frutos se obtuvo con una balanza electrónica marca Ocony, modelo TH-IEK, de $5000.0 \pm 0.1 \mathrm{~g}$ de capacidad. El porcentaje de sólidos solubles totales se determinó con un refractómetro manual marca Atago, modelo $\mathrm{N}-1 \mathrm{a}$, con una escala de $0.0-32.0 \pm 0.2{ }^{\circ}$ Brix. Para la evaluación de firmeza del fruto, se utilizó un penetrómetro marca Chatillon, modelo DPP$100 \mathrm{~N}$, con una capacidad de $100 \pm 1 \mathrm{~N}$. Para la evaluación del $\mathrm{pH}$ se utilizó un medidor electrónico de $\mathrm{pH}$ marca Hanna Instruments, modelo HI 98129, con escala de $0.00-14.00 \pm$ 0.01 .

Se utilizó un diseño experimental irrestricto al azar, donde los cuatro tipos de tomate 
correspondieron a los tratamientos, y los genotipos correspondieron a las repeticiones. Para cada genotipo se sembró una parcela con 8 plantas (2 sacos), y todos los datos se obtuvieron a partir de los frutos totales producidos en dicha parcela. Para cada variable, se realizó un análisis de variancia, y se utilizó la prueba LSD Fisher con una significancia de 5\% para determinar las diferencias entre tratamientos.

\section{Resultados y discusión}

Los tomates que iniciaron cosecha en forma más precoz fueron los tipos 'cherry', uva y 'cocktail' (entre 64.8 y $70.2 \mathrm{ddt}$ ), mientras que el tipo gordo fue significativamente más tardío (76.5 ddt) (figura 1). Esto sugiere una correlación entre esta variable y el peso del fruto, al ser más tardíos los tipos de tomate que presentan frutos con mayor peso. En comparación con los resultados encontrados por otros autores (tabla 2 ), en el presente ensayo se obtuvieron valores intermedios para esta variable en los tipos 'cherry' y gordo, y valores menores en el tipo 'cocktail'.
El número de frutos por racimo fue significativamente mayor en los tomates de frutos de tamaño pequeño (uva y 'cherry') en relación a los tipos 'cocktail' y gordo; asimismo, el número de frutos por racimo del tomate tipo 'cocktail' también fue significativamente mayor que en el caso del tomate tipo gordo (figura 2). Los resultados hallados en el presente estudio fueron similares a los informados por otros autores para los tipos de tomate 'cherry' y gordo (tabla 3).

Los tomates tipo uva, 'cherry' y 'cocktail' presentaron mayoritariamente frutos con sólo 2 lóculos, mientras que el tomate tipo gordo mostró en promedio 6.21 lóculos por fruto (figura 3); las diferencias obtenidas para esta característica entre el tomate tipo gordo y los demás tipos de tomate fueron estadísticamente significativas.

Otros investigadores evaluaron 14 genotipos de tomate tipo gordo, y encontraron entre 3.33 y 8.33 lóculos por fruto (Kumar, y otros, 2006), lo cual coincide con los resultados obtenidos en el presente estudio para ese tipo de tomate.

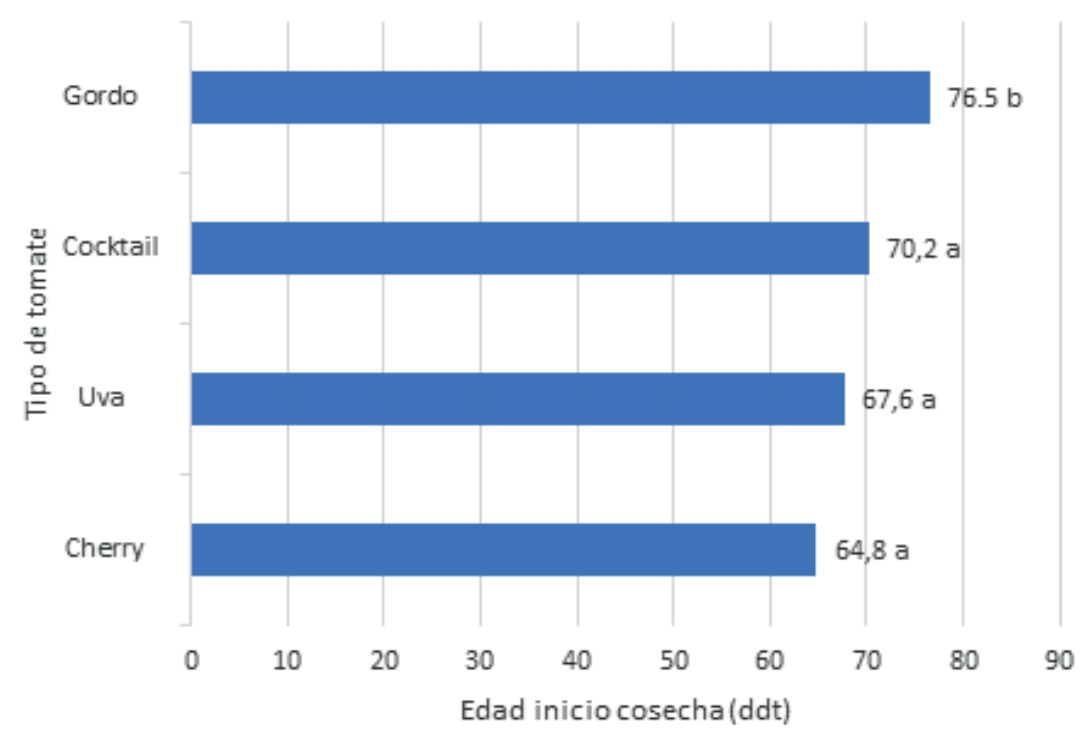

Figura 1. Edad al inicio de la cosecha (ddt), según el tipo de tomate. Nota. Monge y Loría, 2018, a partir de los datos obtenidos. Los tratamientos con una letra en común no presentan diferencias significativas entre ellos, según la prueba LSD Fisher $(p \leq 0.05)$. 
Tabla 2

Edad al inicio de la cosecha, informada en la literatura para producción de tomate en invernadero, según el tipo de tomate

\begin{tabular}{|c|c|c|}
\hline Tipo de tomate & $\begin{array}{l}\text { Edad al inicio de la } \\
\text { cosecha (ddt) }\end{array}$ & Referencia \\
\hline \multirow{7}{*}{ 'Cherry' } & 42 & (Grijalva-Contreras, Robles-Contreras, y Macías-Duarte, 2009) \\
\hline & 45 & (Maboko y Du Plooy, 2008) \\
\hline & 45 & (Rocha, Peil, y Cogo, 2010) \\
\hline & $52-100$ & $\begin{array}{l}\text { (Grijalva-Contreras, Macías-Duarte, Grijalva-Durón, Núñez-Ra- } \\
\text { mírez, y Robles-Contreras, 2014) }\end{array}$ \\
\hline & $76-92$ & (Vargas, y otros, 2017) \\
\hline & 95 & (Herrera, Hurtado-Salazar, y Ceballos-Aguirre, 2015) \\
\hline & 113 & (Mazuela, Acuña, Álvarez, y Fuentes, 2010) \\
\hline ‘Cocktail' & 140 & (Santiago, Mendoza, y Borrego, 1998) \\
\hline \multirow{11}{*}{ Gordo } & 54 & (Gagnon, Mzouri, y Gosselin, 1995) \\
\hline & $64-72$ & (Navarrete y Jeannequin, 2000) \\
\hline & 70 & (Pérez, Albarracín, Moratinos, y Zapata, 2012) \\
\hline & 79 & (Cantliffe, y otros, 2009) \\
\hline & 86 & (Pires, y otros, 2011) \\
\hline & $94-103$ & (Grijalva-Contreras, Macías-Duarte, y Robles-Contreras, 2011) \\
\hline & $99.5-134.5$ & (Santiago, Mendoza, y Borrego, 1998) \\
\hline & $99.7-105.0$ & (Montenegro, 2012) \\
\hline & $105-112$ & (Grijalva-Contreras, Robles-Contreras, y Macías-Duarte, 2009) \\
\hline & 118 & $\begin{array}{l}\text { (Grijalva-Contreras, Macías-Duarte, Valenzuela-Ruiz, y Ro- } \\
\text { bles-Contreras, Productivity and fruit quality in tomatoes varieties } \\
\text { under greenhouse conditions in the Northwest of Mexico, 2004) }\end{array}$ \\
\hline & 135 & (Ardila, Fischer, y Balaguera-López, 2011) \\
\hline
\end{tabular}

Nota. Monge y Loría, 2018, a partir de los datos de la literatura. 


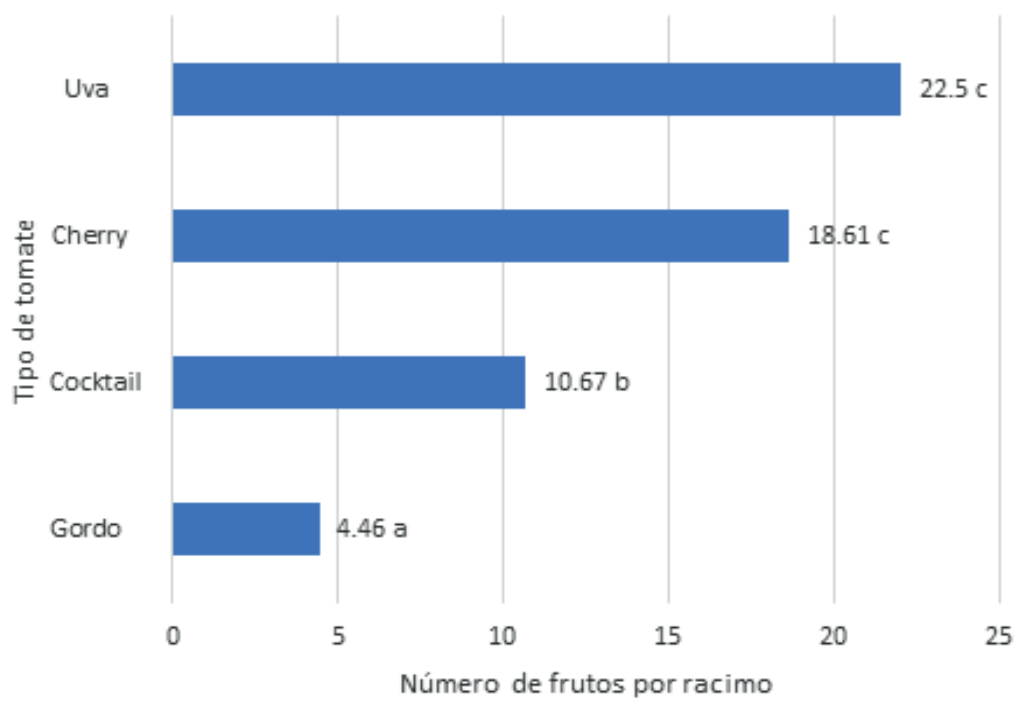

Figura 2. Número de frutos por racimo, según el tipo de tomate. Nota. Elaboración propia, a partir de los datos obtenidos. Los tratamientos con una letra en común no presentan diferencias significativas entre ellos, según la prueba LSD Fisher $(p \leq 0.05)$

\section{Tabla 3}

Número de frutos por racimo, informado en la literatura para producción de tomate en invernadero, según el tipo de tomate

\begin{tabular}{lll}
\hline Tipo de tomate & $\begin{array}{l}\text { Número de frutos } \\
\text { por racimo }\end{array}$ & Referencia \\
\hline
\end{tabular}

4.65 - $15.31 \quad$ (Herrera, Hurtado-Salazar, y Ceballos-Aguirre, 2015)

'Cherry'

$11.52-29.15 \quad$ (Vargas, y otros, 2017)

19.5 (Rocha, Peil, y Cogo, 2010)

Gordo 


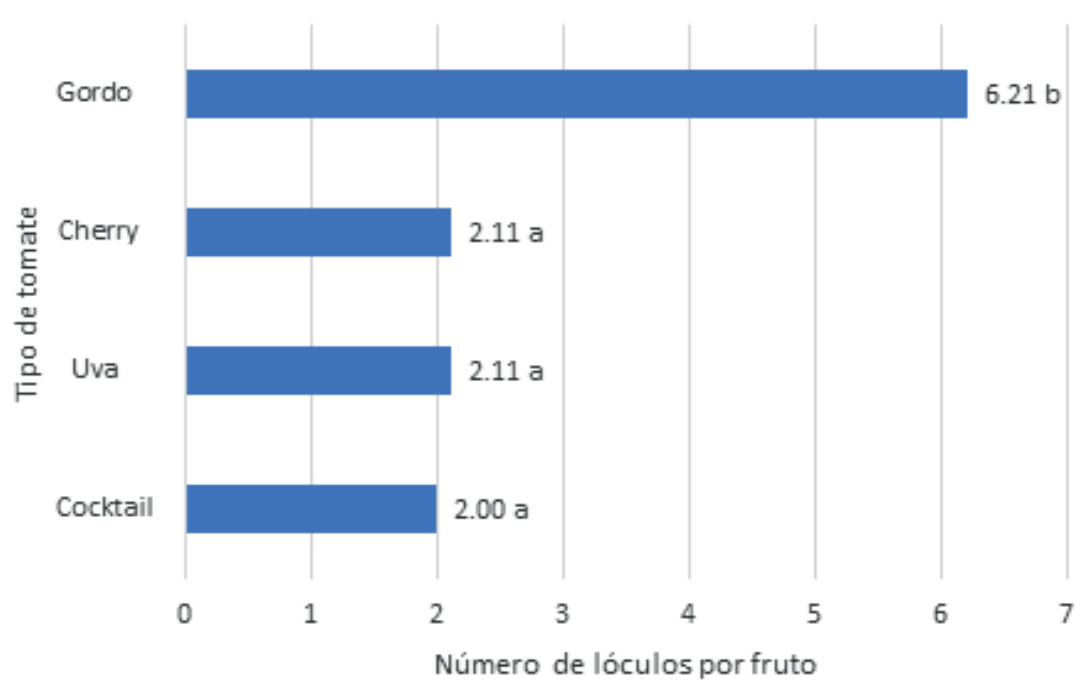

Figura 3. Número de lóculos del fruto, según el tipo de tomate. Nota. Elaboración propia, a partir de los datos obtenidos. Los tratamientos con una letra en común no presentan diferencias significativas entre ellos, según la prueba LSD Fisher $(p \leq 0.05)$.

Con respecto al peso promedio del fruto, no se encontraron diferencias estadísticamente significativas entre los tipos 'cherry', uva y 'cocktail' (entre 12.41 y $24.50 \mathrm{~g} /$ fruto), pero sí entre esos tipos y el tipo gordo (126.80 g/fruto) (figura 4). Los resultados obtenidos coinciden con los informados por otros investigadores para los tomates 'cherry', 'cocktail' y gordo (tabla 4).

En relación al rendimiento, no se presentaron diferencias significativas entre los tipos uva, 'cherry' y 'cocktail' (entre 28.17 y 33.93 ton/ ha), pero sí entre ellos y el tipo gordo (51.18 ton/ha) (figura 5). Los resultados obtenidos en el presente ensayo coinciden con los hallados en otras investigaciones para los tipos 'cherry' y gordo, pero son menores en el caso del tipo 'cocktail' (tabla 5).

En Costa Rica, el precio al consumidor de los tomates tipo 'cherry' es de alrededor de 3000 colones $/ \mathrm{kg}$, mientras que el precio de los tomates gordos es de entre 500 y 1500 colones $/ \mathrm{kg}$, según la época del año (J. Monge-Pérez, datos sin publicar). Esta es una variable a tomar en cuenta, pues se debe relacionar el rendimiento con el precio del producto, para evaluar la rentabilidad económica por área de cada tipo de tomate.

El rendimiento es una cualidad que responde a diversas variables, como el tipo de tomate, el peso del fruto, el ciclo de cultivo, el genotipo, las condiciones ambientales, la presencia de plagas y enfermedades, ladensidad desiembra, y las podas.

En resumen, en los ensayos mostrados en la tabla 5, el rendimiento varió entre 8.60 y 343.0 ton/ha, mientras que en el presente estudio el rendimiento varió entre 28.17 y 51.18 ton/ ha. Una forma de explicar estas diferencias en rendimiento es que en el presente estudio se usó una densidad de siembra de 2.60 plantas/ $\mathrm{m}^{2}$, mientras que en los estudios informados en la literatura las densidades variaron entre 1.89 y 5.30 plantas $/ \mathrm{m}^{2}$. Por otra parte, en el presente ensayo, todas las plantas se podaron a un tallo, pero en los tomates tipo 'cherry', uva y 'cocktail' se recomienda dejar dos o más tallos por planta, para obtener un mayor rendimiento. Además, en el presente estudio el periodo de evaluación fue muy corto (entre 105 y $126 \mathrm{ddt}$ ), mientras que en los ensayos informados en la literatura dicho 


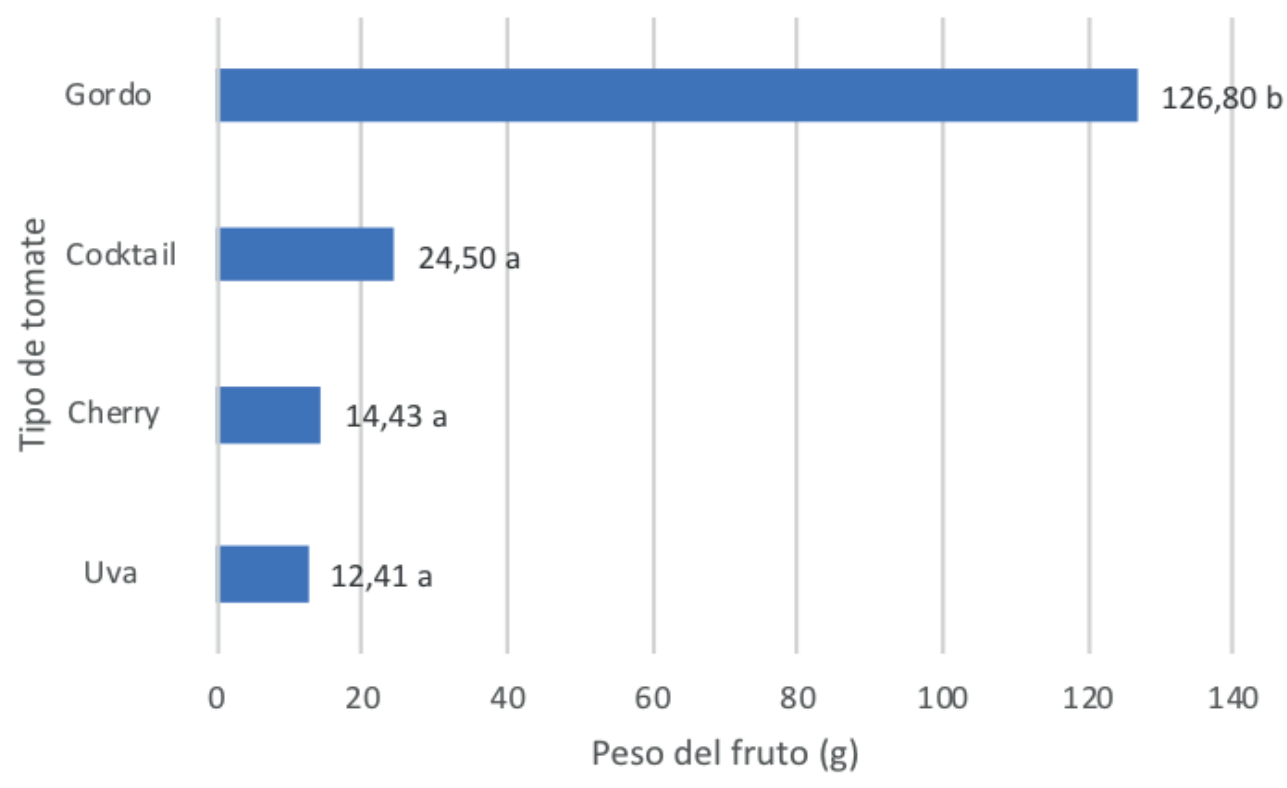

Figura 4. Peso del fruto (g), según el tipo de tomate. Nota. Elaboración propia, a partir de los datos obtenidos. Los tratamientos con una letra en común no presentan diferencias significativas entre ellos, según la prueba LSD Fisher $(p \leq 0.05)$.

Tabla 4

Peso del fruto, informado en la literatura para producción de tomate en invernadero, según el tipo de tomate.

Tipo de tomate $\begin{aligned} & \text { Peso promedio } \\ & \text { del fruto }(\mathrm{g})\end{aligned} \quad$ Referencia

2.57 - 11.88 (Herrera, Hurtado-Salazar, y Ceballos-Aguirre, 2015)

$4.86-13.08 \quad$ (Vargas, y otros, 2017)

$\begin{array}{ccc} & 5-27 & \text { (Causse, Buret, Robini, y Vers } \\ 9.5-13.0 & \text { (Rocha, Peil, y Cogo, 2010) }\end{array}$

13.6-25.7 (Grijalva-Contreras, Macías-Duarte, Grijalva-Durón, Núñez-Ramírez, y Robles-Contreras, 2014)

$23.29-46.35 \quad$ (Sancho, 2013)

'Cocktail' $\quad 31.0 \quad$ (Santiago, Mendoza, y Borrego, 1998)

55.75 (Maboko, Du Plooy, y Chiloane, 2017)

Continúa... 


\begin{tabular}{ccl}
\hline $63.1-149.4$ & (Santiago, Mendoza, y Borrego, 1998) \\
$63.20-93.87$ & (Maboko, Du Plooy, y Chiloane, 2017) \\
$90.2-217.7$ & (Cantliffe, y otros, 2009) \\
$96.5-174.0$ & $\begin{array}{l}\text { (Grijalva-Contreras, Macías-Duarte, Valenzuela-Ruiz, y Ro- } \\
\text { bles-Contreras, Productivity and fruit quality in tomatoes varieties } \\
\text { under greenhouse conditions in the Northwest of Mexico, 2004) }\end{array}$ \\
$108.5-143.3$ & (Shirahige, y otros, 2009) \\
$115-198$ & (Causse, Buret, Robini, y Verschave, 2003) \\
$124.7-258.0$ & (Montenegro, 2012) \\
$138-141$ & (Navarrete y Jeannequin, 2000) \\
$146.51-215.44$ & (Pérez, Albarracín, Moratinos, y Zapata, 2012) \\
$147.6-229.5$ & (Grijalva-Contreras, Macías-Duarte, y Robles-Contreras, 2011) \\
$153.6-224.1$ & (Grijalva-Contreras, Robles-Contreras, y Macías-Duarte, 2009) \\
$200.4-247.6$ & (Grijalva-Contreras, Macías-Duarte, Valenzuela-Ruiz, y Ro- \\
& bles-Contreras, Influence of plant density on yield and quality in \\
& tomatoes under greenhouse conditions in the Northwest on Mexi- \\
& co, 2004) \\
\hline
\end{tabular}

Nota. Monge y Loría, 2018, a partir de los datos de la literatura.

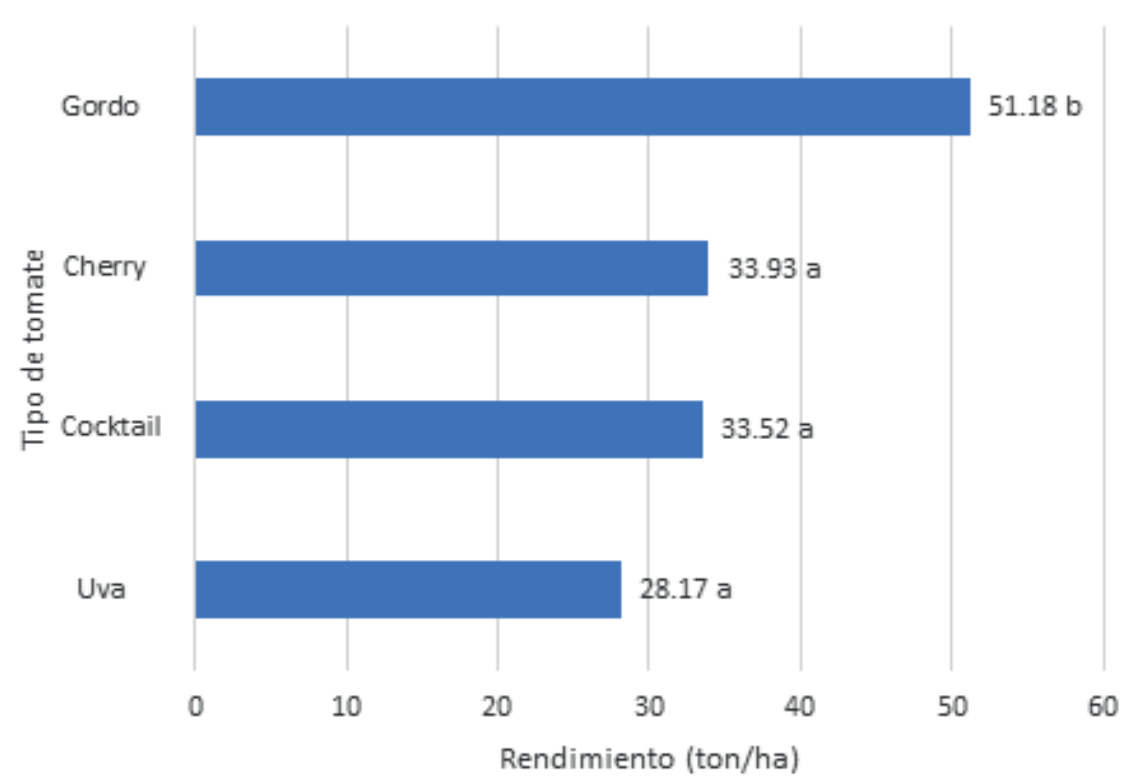

Figura 5. Rendimiento (ton/ha), según el tipo de tomate. Nota. Elaboración propia, a partir de los datos obtenidos. Los tratamientos con una letra en común no presentan diferencias significativas entre ellos, según la prueba LSD Fisher $(p \leq 0.05)$. 
Producción de tomate (Solanum lycopersicum) en invernadero

Tabla 5

Rendimiento informado en la literatura para producción de tomate en invernadero, según el tipo de tomate.

\begin{tabular}{|c|c|c|c|}
\hline $\begin{array}{l}\text { Tipo de } \\
\text { tomate }\end{array}$ & $\begin{array}{l}\text { Rendimiento } \\
\quad \text { (ton/ha) }\end{array}$ & $\begin{array}{c}\text { Ciclo de } \\
\text { cultivo (ddt) }\end{array}$ & Referencia \\
\hline \multirow{7}{*}{ ‘Cherry' } & $8.60-41.66$ & n.d. & (Herrera, Hurtado-Salazar, y Ceballos-Aguirre, 2015) \\
\hline & $32.4-37.9$ & 145 & (Mazuela, Acuña, Álvarez, y Fuentes, 2010) \\
\hline & $37.5-69.7$ & 88 & (Rocha, Peil, y Cogo, 2010) \\
\hline & $48.64-51.57$ & 135 & (Maboko y Du Plooy, 2008) \\
\hline & $56.7-75.3$ & 182 & (Vargas, y otros, 2017) \\
\hline & $118-150$ & 180 & (Grijalva-Contreras, Robles-Contreras, y Macías-Duarte, 2009) \\
\hline & $118-201$ & 249 & $\begin{array}{l}\text { (Grijalva-Contreras, Macías-Duarte, Grijalva-Durón, Núñez-Ramírez, y } \\
\text { Robles-Contreras, 2014) }\end{array}$ \\
\hline \multirow[t]{2}{*}{ 'Cocktail' } & 48.8 & 203 & (Santiago, Mendoza, y Borrego, 1998) \\
\hline & 222 & 125 & (Maboko, Du Plooy, y Chiloane, 2017) \\
\hline \multirow{17}{*}{ Gordo } & $30-120$ & n.d. & (Hernández, 1987) \\
\hline & $52.4-116.0$ & 140 & (Shirahige, y otros, 2009) \\
\hline & $60.75-136.48$ & n.d. & (Maboko, Du Plooy, y Chiloane, 2011) \\
\hline & $70.4-157.5$ & 155 & (Pires, y otros, 2011) \\
\hline & $77.2-121.6$ & 182 & (Montenegro, 2012) \\
\hline & 89 & n.d. & (Campiño y Puerto, 2000) \\
\hline & 96 & n.d. & (Ramírez y Nienhuis, 2012) \\
\hline & $97.2-226.0$ & 203 & (Santiago, Mendoza, y Borrego, 1998) \\
\hline & $107.9-152.3$ & 125 & (Pérez, Albarracín, Moratinos, y Zapata, 2012) \\
\hline & $108-271$ & 183 & (Cantliffe, y otros, 2009) \\
\hline & $147-218$ & n.d. & $\begin{array}{l}\text { (Grijalva-Contreras, Macías-Duarte, Valenzuela-Ruiz, y Robles-Contreras, } \\
\text { Influence of plant density on yield and quality in tomatoes under green- } \\
\text { house conditions in the Northwest on Mexico, 2004) }\end{array}$ \\
\hline & $177-343$ & $247-267$ & (Grijalva-Contreras, Robles-Contreras, y Macías-Duarte, 2009) \\
\hline & $178-343$ & 253 & (Grijalva-Contreras, Macías-Duarte, y Robles-Contreras, 2011) \\
\hline & $192-262$ & 214 & $\begin{array}{l}\text { (Grijalva-Contreras, Macías-Duarte, Valenzuela-Ruiz, y Robles-Contreras, } \\
\text { Productivity and fruit quality in tomatoes varieties under greenhouse con- } \\
\text { ditions in the Northwest of Mexico, 2004) }\end{array}$ \\
\hline & $201-260$ & 125 & (Maboko, Du Plooy, y Chiloane, 2017) \\
\hline & $257.0-305.5$ & 135 & (Ardila, Fischer, y Balaguera-López, 2011) \\
\hline & $301-322$ & n.d. & (Navarrete y Jeannequin, 2000) \\
\hline
\end{tabular}

Nota. Monge y Loría, 2018, a partir de los datos de la literatura. 
periodo varió entre 88 y 267 ddt. Por último, se observa en el cuadro 5 una tendencia a obtener un mayor rendimiento con los tipos de tomate con frutos de mayor peso (especialmente tomates gordos de más de $150 \mathrm{~g} /$ fruto), mientras que en el presente estudio solamente seis genotipos (entre los 17 tomates tipo gordo evaluados) presentaron frutos con un peso promedio superior a $150 \mathrm{~g}$.

La firmeza del fruto presentó diferencias significativas entre los tipos de tomate, con los menores valores para el tipo 'cherry' $(21.34 \mathrm{~N})$, valores intermedios para los tipos uva y 'cocktail' (entre 32.98 y $35.18 \mathrm{~N}$ ), y con los valores más altos para el tipo gordo (47.13 N) (figura 6).

Un fruto maduro de tomate se considera como muy firme, si muestra una firmeza igual o superior a $11 \mathrm{~N}$ (Castellanos, 2009); con este nivel de firmeza, esos frutos ostentan un periodo mayor de vida de anaquel; todos los tipos de tomate evaluados en el presente estudio superaron dicho valor.

En Francia se informó que los tomates gordos presentaron más firmeza del fruto en relación a los tomates 'cherry'; también se halló que, entre los tomates de frutos pequeños, la firmeza mostró una correlación positiva con el peso del fruto; pero esta correlación fue negativa en el caso de los tomates con frutos grandes (Causse, Buret, Robini, y Verschave, 2003). En el presente estudio, igualmente se observó esta tendencia, puesto que los tipos de tomate con frutos más pequeños presentaron la menor firmeza; sin embargo, hay que destacar que los tomates uva mostraron una firmeza significativamente mayor que los tomates 'cherry' y una firmeza similar a los tomates 'cocktail'.

Con respecto a esta característica, en una evaluación entre 11 genotipos de tomate gordo, se halló una firmeza del fruto entre 5.83 y 13.60 N (Zahedi, Ansari, y Eftekhari, 2012). Para un genotipo de tomate 'cherry', otros investigadores encontraron una firmeza del fruto entre 10.88 y 11,18 N (Mazuela, Acuña, Álvarez, y Fuentes, 2010). Por otra parte, en un ensayo con cinco tomates 'cocktail', la firmeza de fruto varió entre 48.75 y $72.95 \mathrm{~N}$ (Sancho, 2013). Todos estos resultados son muy diferentes a los obtenidos en el presente estudio, lo que se puede explicar debido a las diferentes metodologías usadas para evaluar tal característica.

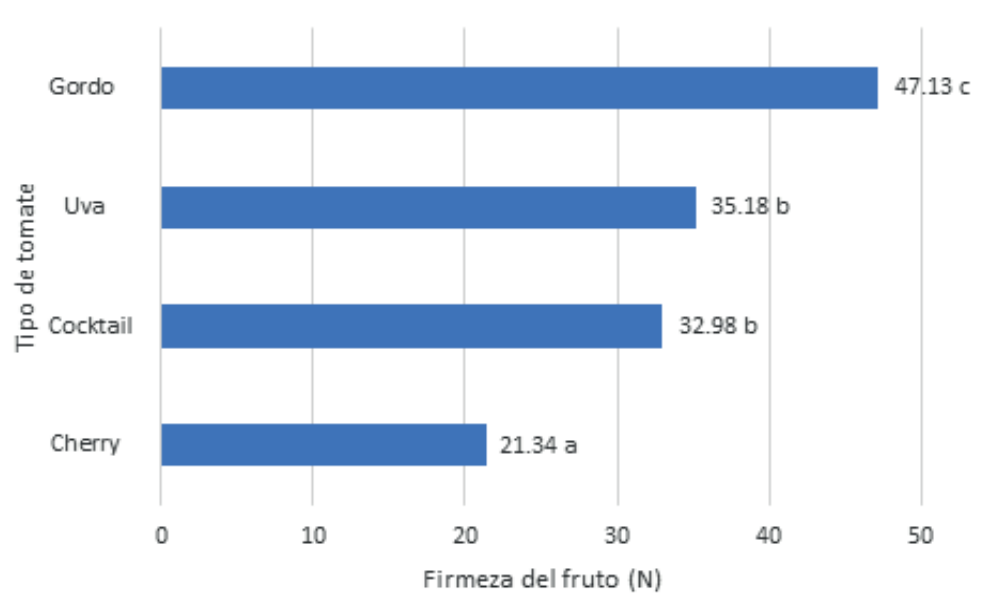

Figura 6. Firmeza del fruto (N), según el tipo de tomate. Nota. Elaboración propia, a partir de los datos obtenidos. Los tratamientos con una letra en común no presentan diferencias significativas entre ellos, según la prueba LSD Fisher $(p \leq 0.05)$. 
El porcentaje de sólidos solubles totales obtenido fue significativamente menor para los tomates tipo gordo (4.41 ${ }^{\circ}$ Brix), y fue significativamente mayor para los tomates tipo uva $\left(8.08^{\circ}\right.$ Brix) y 'cherry' (7.16 'Brix) (figura 7$)$; mientras que el tipo 'cocktail' obtuvo valores intermedios (estadísticamente similar al tipo 'cherry', pero inferior al tipo uva). Los valores obtenidos en el presente estudio fueron similares a los informados por otros autores en el caso de los tomates tipo 'cherry', 'cocktail' y gordo; no obstante, fueron superiores en el caso del tomate tipo uva (tabla 6).

En general, en Costa Rica la mayoría de las variedades comerciales de tomate gordo presenta un ${ }^{\circ}$ Brix entre 4.0 y 5.0 (J. Monge-Pérez, datos sin publicar).

En un fruto maduro de tomate, el porcentaje de sólidos solubles totales se ubica generalmente alrededor de 4.0 a $4.5^{\circ} \mathrm{Brix}$; los frutos con valores superiores a $4.5^{\circ}$ Brix se catalogan como de buen sabor, mientras que los que tienen menos de 4.0 ${ }^{\circ}$ Brix se consideran de baja calidad (Castellanos, 2009). Según este parámetro, en el presente estudio los tomates tipo 'cherry', 'cocktail' y uva se consideran de buen sabor.

Con respecto al $\mathrm{pH}$ del jugo del fruto, el tipo 'cocktail' presentó un valor de 3.73, y este resultado fue significativamente menor en comparación a los obtenidos por los tipos 'cherry', gordo y uva (entre 3.97 y 4.02) (figura 8). Los valores hallados en el presente ensayo para esta variable fueron menores a los informados por otros investigadores para los cuatro tipos de tomate estudiados (tabla 7):

Durante la maduración de los frutos de tomate la acidez desciende, por lo que normalmente el valor de $\mathrm{pH}$ aumenta de 4.0 a 4.5 (Castellanos, 2009). En Brasil se consideran como frutos no ácidos los que presentan un $\mathrm{pH}$ mayor de 4.5 (Shirahige, y otros, 2009); según dicho parámetro, los cuatro tipos de tomate evaluados en la presente investigación se consideran ácidos.

El índice de sabor fue menor para los tipos gordo (70.83) y 'cocktail' (85.50), y esos valores fueron significativamente diferentes con respecto a los tipos 'cherry' (114.07) y uva (131.32) (figura 9).

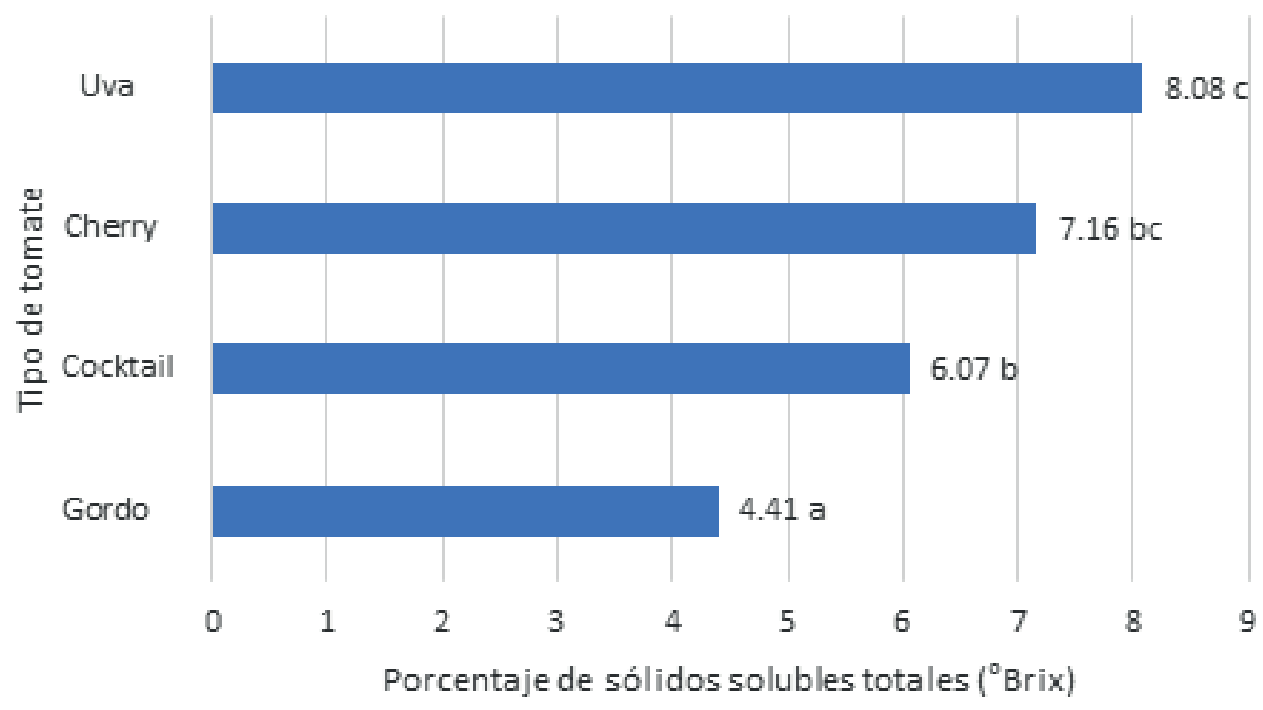

Figura 7. Porcentaje de sólidos solubles totales ( ${ }^{\circ}$ Brix), según el tipo de tomate. Nota. Elaboración propia, a partir de los datos obtenidos. Los tratamientos con una letra en común no presentan diferencias significativas entre ellos, según la prueba LSD Fisher ( $\mathrm{p} \leq 0.05)$. 
Tabla 6

Porcentaje de sólidos solubles totales, informado en la literatura para producción de tomate en invernadero, según el tipo de tomate

\begin{tabular}{lcl}
\hline Tipo de tomate & $\begin{array}{c}\text { Porcentaje de sólidos } \\
\text { solubles totales ('Brix) }\end{array}$ & \multicolumn{1}{c}{ Referencia } \\
\hline \multirow{4}{*}{ 'Cherry' } & $6.0-7.4$ & (Maboko y Du Plooy, 2008) \\
& $6.36-6.80$ & (Vargas, y otros, 2017) \\
& 7.21 & (Causse, Buret, Robini, y Verschave, 2003) \\
& $8.43-9.20$ & (Mazuela, Acuña, Álvarez, y Fuentes, 2010) \\
'Cocktail' & 4.6 & (Santiago, Mendoza, y Borrego, 1998) \\
& $4.82-6.08$ & (Sancho, 2013) \\
& & \\
& $2.4-5.5$ & (Cantliffe, y otros, 2009) \\
& $3.75-4.70$ & (Peet, Harlow, y Larrea, 2004) \\
& $3.9-5.0$ & (Santiago, Mendoza, y Borrego, 1998) \\
Gordo & $4.00-4.73$ & (Shirahige, y otros, 2009) \\
& $4.57-5.10$ & (Pérez, Albarracín, Moratinos, y Zapata, 2012) \\
& $4.62-4.74$ & (Causse, Buret, Robini, y Verschave, 2003) \\
& $5.93-7.49$ & (Pires, y otros, 2011) \\
& & \\
Uva & $6.08-6.32$ & (Pillsbury, Maynard, y Hayes, s.f.) \\
\hline
\end{tabular}

Nota. Monge y Loría, 2018, a partir de los datos de la literatura.

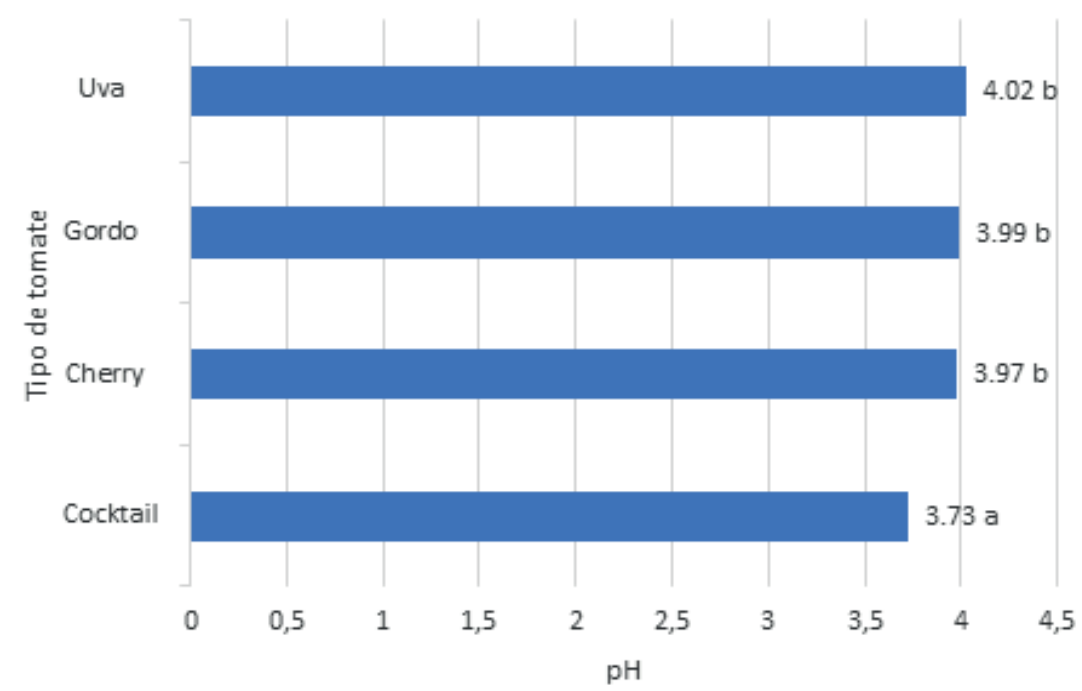

Figura 8. $\mathrm{pH}$ del jugo de los frutos, según el tipo de tomate. Nota. Elaboración propia, a partir de los datos obtenidos. Los tratamientos con una letra en común no presentan diferencias significativas entre ellos, según la prueba LSD Fisher $(p \leq 0.05)$. 
Tabla 7

pH del jugo de los frutos, informado en la literatura para producción de tomate en invernadero, según el tipo de tomate

\begin{tabular}{lcl}
\hline Tipo de tomate & $\begin{array}{c}\mathrm{pH} \text { del jugo de los } \\
\text { frutos }\end{array}$ & \multicolumn{1}{c}{ Referencia } \\
\hline \multirow{2}{*}{ 'Cherry' } & $4.22-4.30$ & (Vargas, y otros, 2017) \\
& 4.26 & (Causse, Buret, Robini, y Verschave, 2003) \\
& & \\
'Cocktail' & $4.61-4.93$ & (Sancho, 2013) \\
& $4.20-4.22$ & (Causse, Buret, Robini, y Verschave, 2003) \\
& $4.2-4.6$ & (Cantliffe, y otros, 2009) \\
& $4.4-4.7$ & (Shirahige, y otros, 2009) \\
Gordo & $4.46-4.63$ & (Pérez, Albarracín, Moratinos, y Zapata, 2012) \\
& & \\
Uva & $4.34-4.55$ & (Pillsbury, Maynard, y Hayes, s.f.) \\
\hline
\end{tabular}

Nota. Monge y Loría, 2018, a partir de los datos de la literatura.

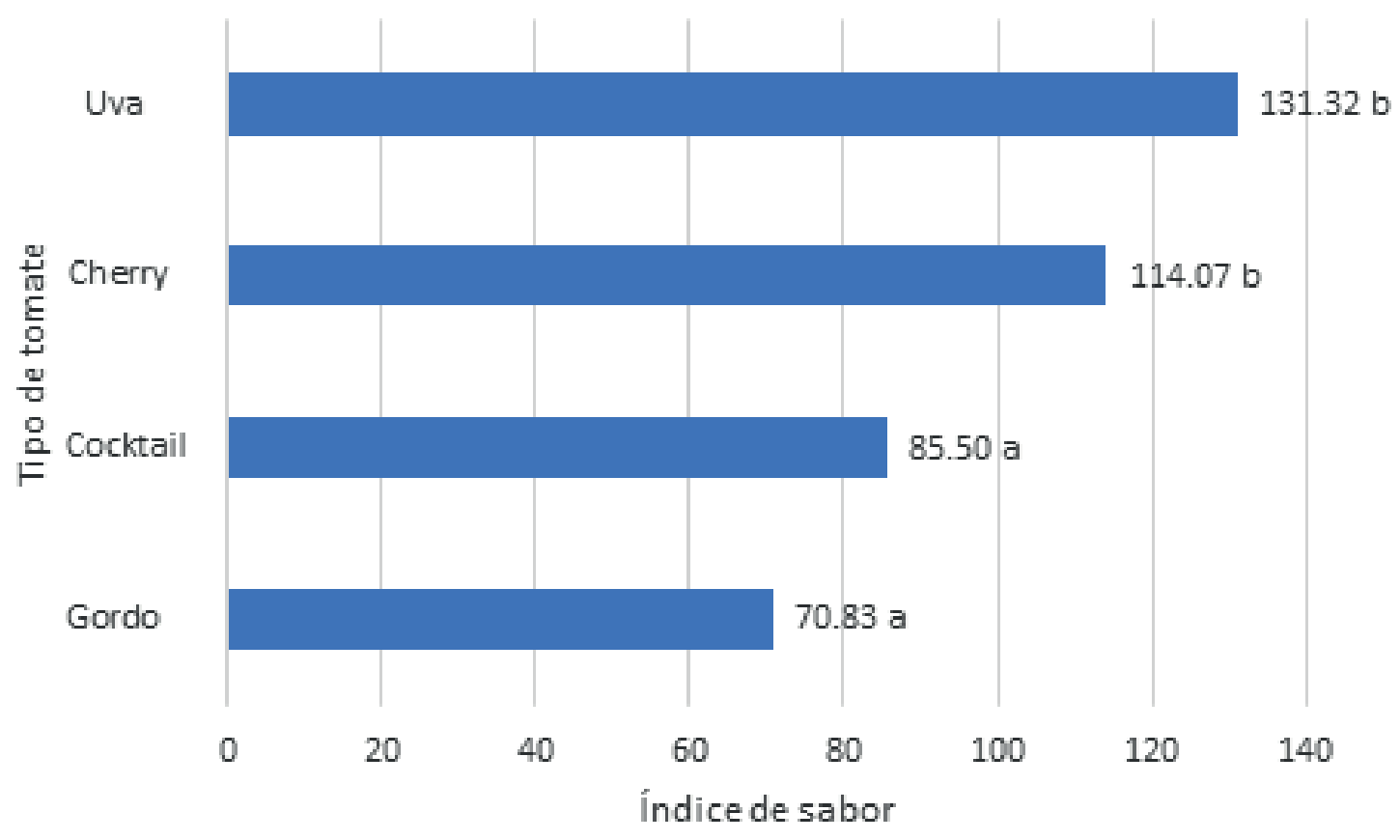

Figura 9. Índice de sabor, según el tipo de tomate. Nota. Elaboración propia, a partir de los datos obtenidos. Los tratamientos con una letra en común no presentan diferencias significativas entre ellos, según la prueba LSD Fisher $(\mathrm{p} \leq 0.05)$. 
El índice de sabor utilizado asume que el mejor sabor se da en los frutos con un alto porcentaje de sólidos solubles totales y una baja acidez (alto $\mathrm{pH}$ ). Otros investigadores han hallado que el consumidor muestra una mayor aceptación de los frutos de tomate con un mayor contenido de azúcares, lo que mejora la percepción de dulzura (Causse, Buret, Robini, y Verschave, 2003; Cebolla-Cornejo, y otros, 2011). Sin embargo, hay que considerar que algunas personas prefieren los tomates con sabor ácido.

Se ha informado que el contenido de sólidos solubles y el $\mathrm{pH}$ son características que se relacionan con la calidad organoléptica del tomate, tales como dulzura o acidez (CebollaCornejo, y otros, 2011). El porcentaje de sólidos solubles totales puede estar relacionado con el contenido de azúcares, pero esta característica dirige en forma importante la preferencia del consumidor solo en los híbridos de frutos pequeños, pero no en los de frutos grandes (Causse, Buret, Robini, y Verschave, 2003; Shirahige, y otros, 2009). Empero, a fin de evaluar la preferencia del consumidos por algún tipo de tomate, se debe recurrir también a la evaluación sensorial (degustación), dado que hay una gran relación entre la textura y el sabor, y los consumidores prefieren frutos con firmeza intermedia (Causse, Buret, Robini, y Verschave, 2003).

En la figura 10, se presentan los resultados de la prueba de degustación. Se destacan los tomates tipo uva con la mejor calificación para esta característica, los cuales presentaron diferencias estadísticamente significativas con respecto a los tomates tipo gordo. El tomate tipo uva también obtuvo más valor en el porcentaje de sólidos solubles totales y en el índice de sabor, por lo que parece que hay una correlación importante entre dichas variables. En otro ensayo, se había identificado que los tomates 'cherry' tienen un mejor sabor en comparación con el resto de tomates, gracias a sus frutos ricos en azúcares y ácidos (Causse, Buret, Robini, y Verschave, 2003).

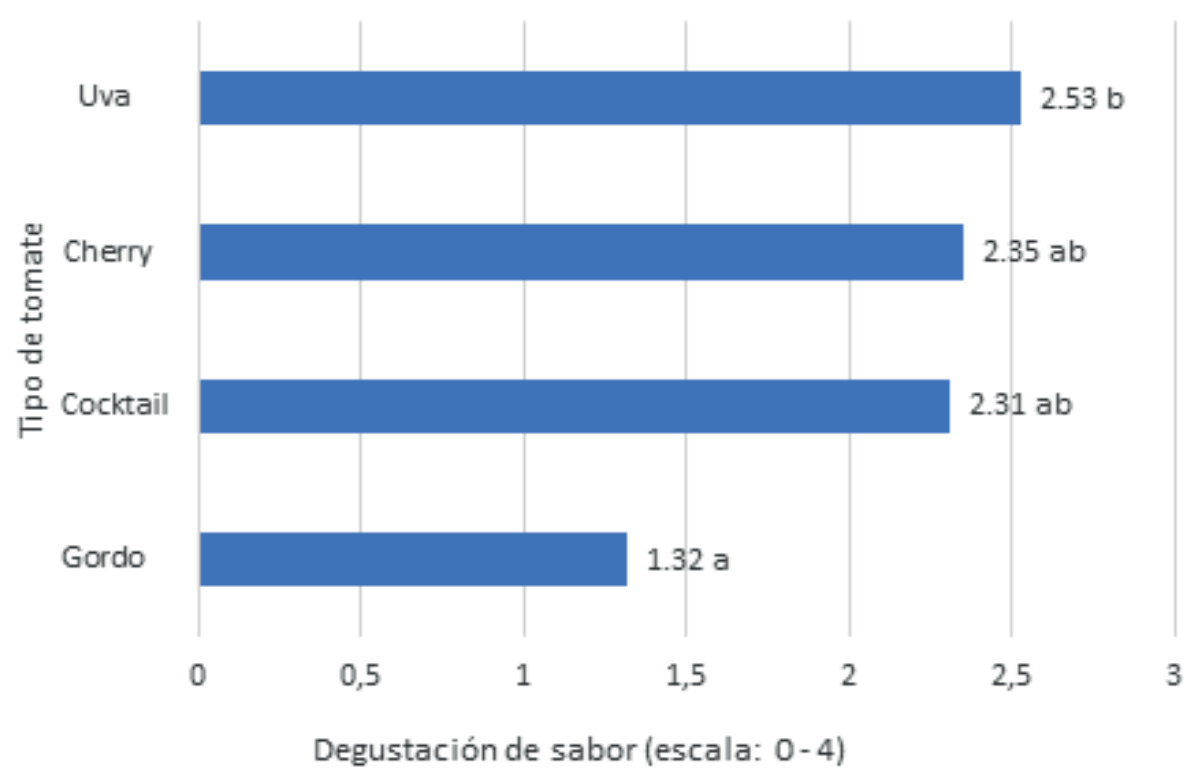

Figura 10. Degustación de sabor (escala: 0 - 4), según el tipo de tomate. Nota. Elaboración propia, a partir de los datos obtenidos. Los tratamientos con una letra en común no presentan diferencias significativas entre ellos, según la prueba LSD Fisher $(p \leq 0.05)$. 


\section{Conclusiones}

Se concluye que, en comparación con los otros tipos de tomate evaluados, los tomates gordos iniciaron la cosecha en forma más tardía, produjeron menos frutos por racimo, presentaron un más lóculos por fruto, tuvieron frutos de mayor peso, produjeron un mayor rendimiento, y sus frutos fueron más firmes, tuvieron un menor porcentaje de sólidos solubles totales, y obtuvieron los peores resultados en la prueba de degustación.

Con respecto a los tomates uva, 'cocktail' y 'cherry', las únicas diferencias significativas entre ellos se presentaron en el número de frutos por racimo, la firmeza del fruto, el porcentaje de sólidos solubles totales, el $\mathrm{pH}$ del jugo del fruto y el índice de sabor.

\section{Agradecimientos}

Los autores agradecen la colaboración de Jorge Díaz, Cristina Arguedas, Carolina Ramírez y Julio Vega en el trabajo de campo; y de Mario Monge en la revisión de la traducción del resumen al idioma inglés. Asimismo, agradecen el financiamiento recibido por parte de la Vicerrectoría de Investigación de la Universidad de Costa Rica (UCR) para la realización de este trabajo.

\section{Referencias}

Alonso, A., García-Aliaga, R., García-Martínez, S., Ruiz, J. J., \& Carbonell-Barrachina, A. A. (2009). Characterization of spanish tomatoes using aroma composition and discriminant analysis. Food Science and Technology International, 15(1), 47-55.

Ardila, G., Fischer, G., \& Balaguera-López, H. E. (2011). Caracterización del crecimiento del fruto y producción de tres híbridos de tomate (Solanum lycopersicum L.) en tiempo fisiológico bajo invernadero. Revista Colombiana de Ciencias Hortícolas, 5(1), 44-56.
Borrego, F., López, A., Fernández, J. M., Muri1lo, M., Rodríguez, S. A., Reyes, A., \& Martínez, J. M. (2001). Evaluación agronómica de tomate (Lycopersicon esculentum M.) en invernadero. Agronomía Mesoamericana, 12(1), 49-56.

Campiño, V. M., \& Puerto, J. E. (2000). Evaluación de cinco materiales híbridos de tomate bajo condiciones de invernadero en el Municipio de Quipile, Cundinamarca. Santa Fe de Bogotá, Colombia: Facultad de Agronomía, Universidad Nacional de Colombia.

Cantliffe, D. J., Shaw, N. L., Sargent, S. A., Simms, C., Berry, A., Kan, E. L., . . . Scott, J. W. (2009). The determinate 'Tasti-Lee' tomato competes with indeterminate, greenhouse cultivars for yield, fruit quality, and sensory analysis when produced hydroponically. Proceedings of the Florida State Horticultural Society, 122, 275-280.

Castellanos, J. Z. (2009). Manual de producción de tomate en invernadero. Celaya, Guanajuato, México: Intagri S. C.

Causse, M., Buret, M., Robini, K., \& Verschave, P. (2003). Inheritance of nutritional and sensory quality traits in fresh market tomato and relation to consumer preferences. Journal of Food Science, 68(7), 2342-2350.

Cebolla-Cornejo, J., Roselló, S., Valcárcel, M., Serrano, E., Beltrán, J., \& Nuez, F. (2011). Evaluation of genotype and environment effects on taste and aroma flavor components of spanish fresh tomato varieties. Journal of Agricultural and Food Chemistry, 59, 2440-2450.

Fernández-Ruiz, V., Sánchez-Mata, M. C., Cámara, M., Torija, M. E., Chaya, C., Galiana-Balaguer, L., . . . Nuez, F. (2004). Internal quality characterization of fresh tomato fruits. Hortscience, 39(2), 339-345. 
Gagnon, S., Mzouri, M., \& Gosselin, A. (1995). Effects of cultivars and culture systems on yield and postharvest quality of greenhouse tomato. Hortscience, 30(4), 847.

Grijalva-Contreras, R. L., Macías-Duarte, R., \& Robles-Contreras, F. (2011). Comportamiento de híbridos de tomate bola en invernadero bajo condiciones desérticas del noroeste de Sonora. Tropical and Subtropical Agroecosystems, 14, 675-682.

Grijalva-Contreras, R. L., Macías-Duarte, R., Grijalva-Durón, S. A., Núñez-Ramírez, F., \& Robles-Contreras, F. (2014). Productividad de cultivares de tomate cherry bajo condiciones de invernadero en el noroeste de Sonora. Biotecnia, 16(2), 27-30.

Grijalva-Contreras, R. L., Macías-Duarte, R., Valenzuela-Ruiz, M., \& Robles-Contreras, F. (2004). Influence of plant density on yield and quality in tomatoes under greenhouse conditions in the Northwest on Mexico. Hortscience, 39(4), 801.

Grijalva-Contreras, R. L., Macías-Duarte, R., Valenzuela-Ruiz, M., \& Robles-Contreras, F. (2004). Productivity and fruit quality in tomatoes varieties under greenhouse conditions in the Northwest of Mexico. Hortscience, 39(4), 804.

Grijalva-Contreras, R. L., Robles-Contreras, F., \& Macías-Duarte, R. (2009). Híbridos de tomate para la producción en invernadero en el noroeste de Sonora. Hermosillo, Sonora, México: Instituto Nacional de Investigaciones Forestales, Agrícolas y Pecuarias.

Hernández, F. (1987). Evaluación de la adaptabilidad de 5 cultivares de tomate (Lycopersicon esculentum Mill) en la zona de Guaduas, Cundinamarca. Santa Fe de Bogotá, Colombia: Facultad de Agronomía, Universidad Nacional de Colombia.
Herrera, H. d., Hurtado-Salazar, A., \& Ceballos-Aguirre, N. (2015). Estudio técnico y económico del tomate tipo cereza élite (Solanum lycopersicum L. var. cerasiforme) bajo condiciones semicontroladas. Revista Colombiana de Ciencias Hortícolas, 9(2), 290-300.

IPGRI. (1996). Descriptores para el tomate (Lycopersicon spp.). Roma, Italia: International Plant Genetic Resources Institute. Retrieved from http://pdf.usaid.gov/pdf_docs/ PNACH866.pdf

Kumar, R., Mishra, N. K., Singh, J., Rai, G. K., Verma, A., \& Rai, M. (2006). Studies on yield and quality traits in tomato (Solanum lycopersicon (Mill.) Wettsd.). Vegetable Science, 33(2), 126-132.

Lorca, M. (1997). Evaluación agronómica de 15 híbridos comerciales de tomate (Lycopersicon esculentum Mill.) indeterminado para consumo fresco bajo invernadero. Santiago, Chile: Facultad de Ciencias Agrarias y Forestales, Universidad Iberoamericana de Ciencias y Tecnología.

Maboko, M. M., \& Du Plooy, C. P. (2008). Effect of pruning on yield and quality of hydroponically grown cherry tomato (Lycopersicon esculentum). South African Journal of Plant and Soil, 25(3), 178-181.

Maboko, M. M., Du Plooy, C. P., \& Chiloane, S. (2011). Effect of plant population, fruit and stem pruning on yield and quality of hydroponically grown tomato. African Journal of Agricultural Research, 6(22), 5144-5148.

Maboko, M. M., Du Plooy, C. P., \& Chiloane, S. (2017). Yield of determinate tomato cultivars grown in a closed hydroponic system as affected by plant spacing. Horticultura Brasileira, 35(2), 258-264.

Mazuela, P., Acuña, L., Álvarez, M., \& Fuentes, A. (2010). Producción y calidad de un 
tomate cherry en dos tipos de invernadero en cultivo sin suelo. Idesia, 28(2), 97-100.

Monge-Pérez, J. E. (2014). Caracterización de 14 genotipos de tomate (Lycopersicon esculentum Mill.) cultivados bajo invernadero en Costa Rica. Tecnología en Marcha, 27(4), 58-68.

Monge-Pérez, J. E. (2015). Evaluación de 60 genotipos de tomate (Lycopersicon esculentum Mill.) cultivados bajo invernadero en Costa Rica. Intersedes, 16(33), 84-122.

Monge-Pérez, J. E. (2016). Evaluación preliminar de 201 genotipos de ocho diferentes hortalizas (berenjena, chile dulce, zucchini, ayote, sandía, pepino, tomate y melón) cultivados bajo invernadero en Costa Rica. En E. Solano, La investigación en Guanacaste II (pp. 277-300). San José, Costa Rica: Nuevas Perspectivas.

Montenegro, V. H. (2012). Evaluación de la aclimatación de veinte y dos cultivares de tomate (Lycopersicon esculentum Mill), bajo invernadero, en Chugllin, cantón Chambo, Provincia Chimborazo. Riobamba, Ecuador: Escuela de Ingeniería Agronómica, Facultad de Recursos Naturales, Escuela Superior Politécnica de Chimborazo.

Moricz, M. (s.f.). Investigations to the fruit quality and consumer acceptance of Heirloom tomato varieties. Recuperado de: http://labmath.eu

Navarrete, M., \& Jeannequin, B. (2000). Effect of frequency of axillary bud pruning on vegetative growth and fruit yield in greenhouse tomato crops. Scientia Horticulturae, 86, 197-210.

Peet, M. M., Harlow, C. D., \& Larrea, E. S. (2004). Fruit quality and yield in five small-fruited greenhouse tomato cultivars under high fertilization regime. Acta Horticulturae, 659, 811-818.
Pérez, M. B., Albarracín, M., Moratinos, H., \& Zapata, F. (2012). Rendimiento y calidad de fruto en cuatro cultivares de tomate (Solanum lycopersicum L.) bajo condiciones protegidas. Revista Facultad Agronomía (LUZ), 29, 395-412.

Pillsbury, L., Maynard, E., \& Hayes, K. (s.f.). Chemical, physical and sensory properties of four grape tomato varieties. Recuperado de: https://www2.ag.purdue.edu/hla/fruitveg/ Documents/pdf/reports/grapetomato_ in_04.pdf

Pires, R. C., Furlani, P. R., Ribeiro, R. V., Junior, D. B., Sakai, E., Lourenção, A. L., \& Neto, A. T. (2011). Irrigation frequency and substrate volume effects in the growth and yield of tomato plants under greenhouse conditions. Scientia Agricola, 68(4), 400-405.

Ramírez, C., \& Nienhuis, J. (2012). Cultivo protegido de hortalizas en Costa Rica. Tecnología en Marcha, 25(2), 10-20.

Rocha, M. d., Peil, R. M., \& Cogo, C. M. (2010). Rendimento do tomate cereja em função do cacho floral e da concentração de nutrientes em hidroponia. Horticultura Brasileira, 28(4), 466-471.

Sancho, M. L. (2013). Características poscosecha de cinco genotipos de tomate (Lycopersicon esculentum Mill.), en una localidad de clima templado (Estados Unidos) comparado con una localidad de clima tropical. Santa Clara, San Carlos, Costa Rica: Sede Regional de San Carlos, Instituto Tecnológico de Costa Rica.

Santiago, J., Mendoza, M., \& Borrego, F. (1998). Evaluación de tomate (Lycopersicon esculentum, Mill) en invernadero: criterios fenológicos y fisiológicos. Agronomía Mesoamericana, 9(1), 59-65.

Shirahige, F. H., Melo, P. C., Jacomino, A. P., Melo, A. M., Purquerio, L. F., \& Roquejani, 
M. S. (2009). Yield and qualitative characterization of fresh market tomato hybrids of Italian and Santa Cruz types. Acta Horticulturae, 821, 81-88.

Vargas, P. F., Duarte, L. S., Silva, E. H., Zecchini, A. C., Soares, R. S., \& Godoy, L. J. (2017). Performance of mini-tomato hybrids in different training systems with different num- ber of stems. Horticultura Brasileira, 35(3), 428-433.

Zahedi, S. M., Ansari, N. A., \& Eftekhari, S. A. (2012). Investigation of yield and adaptation of ten selected genotypes of tomato under subtropical climate conditions (Ahvaz). Journal of Food, Agriculture \& Environment, 10(1), 782-786. 